\title{
Nanostructured gas diffusion layer to improve direct oxygen reduction reaction in Air-Cathode Single-Chamber Microbial Fuel Cells
}

\author{
Giulia Massaglia' ${ }^{1,2 *}$, Eve Verpoorten ${ }^{1,2}$, Candido F. Pirri ${ }^{1,2}$, and Marzia Quaglio ${ }^{1,2}$ \\ ${ }^{1}$ Department of Applied Science and Technology, Politecnico of Turin, 10129, Corso Duca degli Abruzzi 29, Italy \\ ${ }^{2}$ Center for Sustainable Future and Technologies, Italian Institute of Technology, 10100, Via Livorno 60, Italy
}

\begin{abstract}
The aim of this work is the development of new nanostructured-gas-diffusion-layer (GDL) to improve the overall behaviour of Air-Cathode Single-Chamber-Microbial-Fuel-Cells (SCMFCs). The design of new nanostructured-GDL allowed exploiting all nanofibers 'intrinsic properties, such as high surface ratio to volume, high porosity, achieving thus a good oxygen diffusion into the proximity of catalyst layer, favouring thus the direct oxygen-reduction-reaction (ORR). Nanostructured-GDLs were prepared by electrospinning process, using a layer-by-layer deposition to collect 2 nanofibers' mats. The first layer was made of cellulose nanofibers able to promote oxygen diffusion into SCMFC. The second layer, placed outwards, was based on polyvinyl-fluoride (PVDF) nanofibers to prevent the electrolyte leakage. This nanostructured-GDL plays a pivotal role to improve the overall performance of Air-Cathode-SCMFCs. A maximum current density of $20 \mathrm{~mA} \mathrm{~m}^{-2}$ was obtained, which is higher than the one reached with commercialGDL, used as reference material. All results were analysed in terms of energy recovery parameter, defined as ratio of generated power integral and the internal volume of devices, evaluating the overall SCMFC performance. SCMFCs with a nanostructured-GDL showed an energy recovery equal to $60.83 \mathrm{~mJ} \mathrm{~m}^{-3}$, which was one order of magnitude higher than the one obtained with commercial-GDL, close to $3.92 \mathrm{~mJ} \mathrm{~m}^{-3}$.
\end{abstract}

\section{Introduction}

Renewable energy sources play a pivotal role to ensure the transition from carbon-based economies towards sustainable human development [1]. Among all possible renewable energy sources, like wind, solar, hydro-and geo-thermal and biomass, microbial fuel cells (MFCs) have gained great interest in recent years since they are able to combine the power production with some processes like water treatments, bio-remediation and sensing, obtained by involving a particular class of bacteria into MFCs [2-3]. In general, MFCs are bioelectrochemical devices that directly transduce the chemical energy, entrapped into organic matter, into energy one thanks to the metabolic activity of a particular kind of bacteria, named exo-electrogenic bacteria [4-6]. These microorganisms, indeed, are able to release all produced electrons outside their cells to anode electrode surfaces [4-6]. This kind of bacteria proliferated onto the anode surfaces, leading thus to the formation of a biofilm, whose exocellular electron transfer ensured the power output production. In the present work, an open-air cathode configuration MFCs is used, where direct oxygen reduction reaction (ORR) occurred into the cathodic compartment [7]. As investigated in several works in the literature, the optimization of cathode electrodes, with the main purpose to improve the diffusion layer, which plays a crucial role into the diffusion of oxygen from outside into the devices and apply a proper catalyst layer able to ensure the achievement of ORR, is mandatory. In this context, the activation losses at the cathode are minimized and the overall MFCs performance is improved [8-9]. The catalyst layer must provide a number of electrons that are as close as possible to the theoretical value of 4 , favouring the direct ORR and avoiding the intermediate 2-electrons reduction reaction, which releases $\mathrm{H} 2 \mathrm{O} 2$, a toxicant for microorganisms [8-9]. A catalyst layer based on platinum results to be the best performing class of catalyst for this reaction [9]. On the contrary, the common gas diffusion layer (GDL) is composed by polytetrafluoroethylene (PTFE), that is deposited onto carbon-based electrode. PTFE ensures the oxygen diffusion into the cathodic compartment, avoiding/reducing as much as possible the electrolyte leakage. The aim of the present work is the development of a new nanostructured-gas-diffusion-layer (GDL) to improve the overall behaviour of Air-Cathode SingleChamber-Microbial-Fuel-Cells (SCMFCs). The design of new nanostructured-GDL allowed exploiting all nanofibers 'intrinsic properties, such as high surface ratio to volume, high porosity and light weight, achieving thus a good oxygen diffusion into the proximity of catalyst layer, ensuring and favouring the direct oxygen reduction reaction (ORR). Nanostructured-GDLs were prepared by electrospinning process, using a layer-by-layer deposition to collect 2 different nanofibers' mats. The first layer was made of cellulose nanofibers that play a crucial role to promote oxygen diffusion into SCMFC. The second layer, placed outwards, was based on polyvinyl-fluoride (PVDF) nanofibers with the main purpose to prevent the electrolyte leakage, while allowing oxygen free to flow. Cellulose nanofibers were obtained starting from a polymeric solution of cellulose-acetate (CA). To employ final cellulose nanofibers, as fabricated-CA nanofibers were hydrolysed in $0.05 \mathrm{~mol} / \mathrm{L} \mathrm{NaOH} /$ ethanol solution for $24 \mathrm{~h}$ at room temperature, followed by thoroughly washing with double-distilled water until the supernatant reached neutral. Both of 2 nanostructured layers were

\footnotetext{
* Corresponding author: giulia.massaglia@polito.it
} 
directly deposit onto carbon paper. Onto the inner side of carbon paper electrode, a catalyst layer made of $\mathrm{Pt} / \mathrm{C}$ was applied to ensure the direct ORR. To demonstrate how nanostructured GDL can improve the overall performance of Air-Cathode-SCMFCs, this cathode electrode was applied into the devices and compared with the one that presents a commercial GDL, made of polytetrafluoroethylene (PTFE). A maximum current density of $35.71 \mathrm{~mA} \mathrm{~m}-2$ was obtained, which is higher than the one reached with commercial-GDL, used as reference material. All reached results were analysed in terms of the energy recovery parameter, defined as the ratio of the generated power integral and the internal volume of the devices, evaluating the overall SCMFC performance. SCMFCs with a nanostructured-GDL showed an energy recovery equal to $60.83 \mathrm{~mJ} \mathrm{~m}-3$, which was one order of magnitude higher than the one obtained with commercial-GDL, close to $3.92 \mathrm{~mJ} \mathrm{~m}-3$. All these latter results open the doors to design the entire nanostructured cathode electrode in SCMFCs. The nanostructured cathode electrode may be done by carbon nanofibers doped with nitrogen (N-CNFs), which play a pivotal role as promising catalyst layer for direct ORR as demonstrated in our previous work [11] and can be employed as a carbon backbone to ensure the electron transfer produced and released by microorganisms into the anode compartment.

\section{Materials and Methods}

\subsection{Materials and nanofibers synthesis}

The layer-by layer deposition is ensured by implementing the electrospinning process (NANON 01A electrospinning apparatus MECC, LTD). One of the great advantages of this technology, indeed, consist into ability to ensure a layer-by layer deposition without the using of binder to ensure the connection between dried nanofiber mats and $\mathrm{CP}$ material. In particular, two different layers were obtained: $i$ ) the first layer is made of cellulose nanofibers, obtained by a starting polymeric solution based on cellulose acetate $(\mathrm{Mw}=$, Sigma Aldrich) dissolved into N-N DMF (assay 99.8\%, Sigma Aldrich); ii) the second layer, on the contrary, is composed by $2 \mathrm{~g}$ of polyvinyl fluoride (PVDF, $\mathrm{Mw}=$, Sigma Aldrich) dissolved into a mixture of N-N DMF and acetone with a volume ratio of $1: 1$. During the electrospinning process, the working voltage between the needle and planar counter electrode is fixed at $26 \mathrm{kV}$ with a flow rate of $0.5 \mathrm{~mL} \mathrm{~h}-1$. The working distance between the needle and substate is equal to $15 \mathrm{~cm}$. After the deposition of first layer made of cellulose acetate nanofibers must be properly hydrolysed with the main purpose to obtain a final mat of cellulose nanofibers. As fabricated-CA nanofibers were hydrolysed in $0.05 \mathrm{~mol} / \mathrm{L}$ $\mathrm{NaOH} /$ ethanol (Sigma Aldrich) solution for $24 \mathrm{~h}$ at room temperature, followed by thoroughly washing with double-distilled water until the supernatant reached neutral. After this post-process treatment, a deposition of
PVDF nanofibers is achieved to prevent possible leakage of electrolyte solution.

\subsection{SCMFCs architecture and operation}

As deeply used in our previous work [10-11], a squared shape open-air cathode SCMFCs is used during the whole experiment. The devices 'case is realized by $3 \mathrm{D}$ printer (OBJET 30]. SCMFCs are composed by 3 different compartments and the devices are membrane-less cell with electrolyte in common between anode and cathode. The total internal volume is equal to $12.5 \mathrm{~mL}$ and both anode and cathode electrodes show a geometric surface area close to $5.76 \mathrm{~cm} 2$. The anode electrodes is a carbon based materials, carbon paper (CP, purchased from Fuel Cell Earth, USA), on which a biofilm is formed. In the present work, all anode electrodes have been obtained from previous experiments, conducted in our laboratories. In this scenario, microbial biofilm is formed on anodes surfaces. The cathode electrodes are modified by applying a catalyst layer based on platinum $(0.5 \mathrm{mg} \mathrm{cm}-2$ of $\mathrm{Pt} / \mathrm{C}$ obtained by Sigma Aldrich) and $5 \mathrm{wt} \%$ of Nafion (Sigma Aldrich), as discussed in the literature [9]. Moreover, on the inner side of CP a gas diffusion layer is deposited. The commercial GDL, composed by PTFE layer, was directly compared with the new designed nanostructured-GDL. The electrolyte is a water-based solution containing $12 \mathrm{mM}$ of sodium acetate, used as organic matter, and other compounds $(5.8 \mathrm{mM}$ of ammonium chloride and phosphate buffer saline solution (PBS) [10-12]) suitable for the preservation of metabolic activity of microorganisms. Titanium wires were used to ensure a good electrical contact and both anode and cathode were connected with a multichannel data acquisition unit (Agilent 34972 A). an external load of $1 \mathrm{k} \Omega$ is applied. Moreover, during the whole experiments, a fed bath mode is implemented to substitute the old electrolyte with the new one. According to this method, the replacement of electrolyte is carried out when voltage reaches a values close to $0 \mathrm{~V}$. all the experiments were conducted in duplicate.

\subsection{Characterizations and measurements}

Field Emission Scanning Electron Microscopy (FESEM, Supra), operating from $5 \mathrm{kV}$ to $10 \mathrm{kV}$, is used to evaluate the morphological properties of nanostructured gas diffusion layer (GDL). In order to evaluate how nanostructured-GDL can affect SCMFCs performance, polarization curves are defined through Linear Sweep Voltammetry (LSV) performed by using Palmsens potentiostat (Palmsens4, Netherlands)). LSV characterizations performed at the end of experimental study. Moreover, in the present work LSV characterization is provided by implementing a voltage range from open circuit to a short circuit with a rate of $0.1 \mathrm{mV} \mathrm{s}-1$. The catalytic properties of the diverse cathodes were assessed through electrochemical impedance spectroscopy (EIS), employing a Palmsens potentiostat. For EIS, the sinusoidal signal had an 
amplitude of $25 \mathrm{mV}$ and the frequency was spanned between $150 \mathrm{kHz}$ and $200 \mathrm{mHz}$; the fixed resistor method [12] (100 $\Omega$ ) was employed. All experimental data were fitted by defining the equivalent circuit, as reported in in Scheme 1, in order to quantitatively evaluate the electrical parameters; R1 represent the series resistance (accounting for electrolyte and wiring resistances), R2 and R3 denote the charge transport (in the electrode) and the charge transfer (at the electrode/electrolyte interface) resistances, respectively. Due to porous nature of catrhode electrodes, constant phase elements, Q1 and Q2 are used to model the corresponding double layer capacitances [12]). Low frequency feature, corresponding to the species diffusion, was modelled by Warburg element, as included in the fitting.

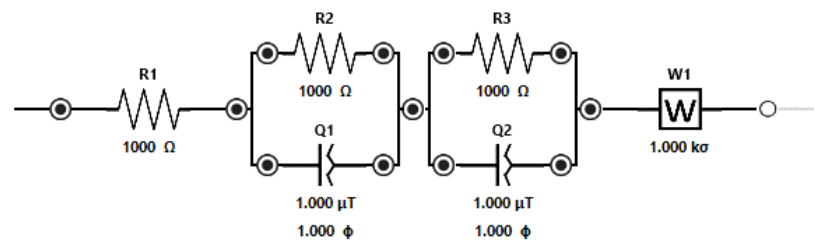

Scheme 1. Equivalent circuit used to fit the impedance spectra.

\section{Results and Discussion}

\subsection{Morphological properties of nanostrucutred- GDL}

The morphological properties of nanostructured-GDL is reported in Fig. 1a). FESEM images highlight the pore distribution in this samples. Indeed nanostructured-GDL are characterized by pores with dimensions in the range of few micrometres, leading thus to exhibit a higher surface area to volume ratio than the one obtained with commercial gas diffusion layers (see Fig. 1b) These intrinsic properties of nanofibers play a pivotal role into the enhancement of oxygen diffusion inside the devices, improving thus direct ORR.
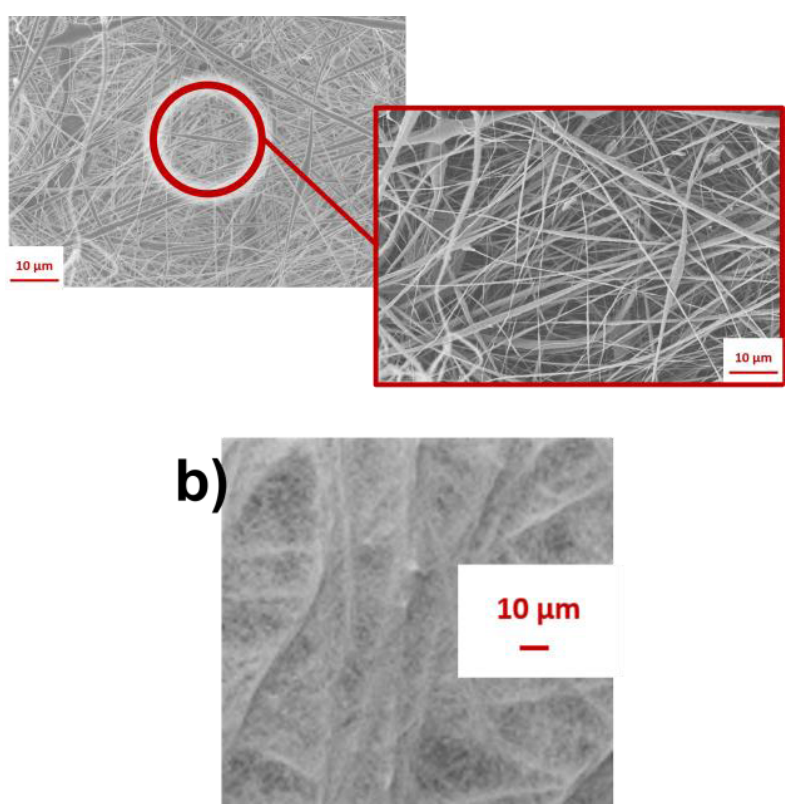

Fig. 1. a) Morphological properties of nanostructured-GDL, confirming high porosity of samples, as highlighted from higher magnification, underlined by red box. b) morphological properties of commercial GDL applied onto carbon paper CP. b) commercial carbon paper with commercial gas diffusion layer

\subsection{SCMFCs performance}

As previously described, at the beginning of experiments, anodes were obtained by preceding experiments, leading thus to employ a biofilm formation onto all anode surfaces. For all cathode electrodes, a catalyst layer based on platinum is applied, while nanostructured-GDL and commercial GDL were compared, to evaluate how can affect the overall performance of SCMFCs. Fig.2. represents the current density trends over time. Nanostructured-GDL reached a maximum current density equal to $(35.7 \pm 2.3) \mathrm{mA} \mathrm{m}-2$, which is double of the one reached with commercial GDL (maximum current density close to $(15.22 \pm 0.21) \mathrm{mA} \mathrm{m}-2)$, used as reference material. Since the anodic compartments of both kinds of devices are nominally identical, the differing performance of the SCMFCs can be directly attributed to the use of diverse GDLs applied onto cathode electrodes. 


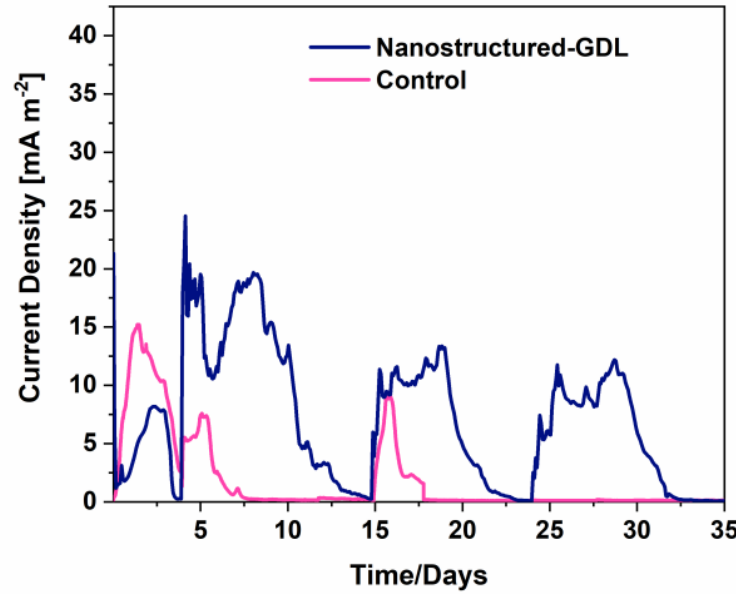

Fig. 2. Comparison of average current density trend of SCMFCs with nanostructured-GDL and current density trend reached by SCMFCs with commercial GDL, defined as reference cathode electrode.

In Fig.3.a) LSV curves, related to both GDLs, are reported. It is possible to notice that the maximum power density with nanostructured-GDL was up to the double of the ones reached by SCMFCs with commercial GDL, defined as control device for the whole experiment. Both of SCMFCs devices reach a similar open circuit voltage (OCV) close to $0.4 \mathrm{~V}$, while SCMFCs with nanostructured-GDL achieved a higher short circuit current that the one obtained with control material. Since nanostructured-GDL can affect and favour the direct ORR, ensuring the best oxygen diffusion into SCMFCs, the variation of total cathodic resistance over time was investigated through EIS. Typical Nyquist plots are represented in Fig.3b), comparing nanostructured-GDL and commercial one. The curves obtained by fitting procedure are overlaid on the experimental data (see Fig.3b). Table 1, on the contrary, summarized all resistance values.
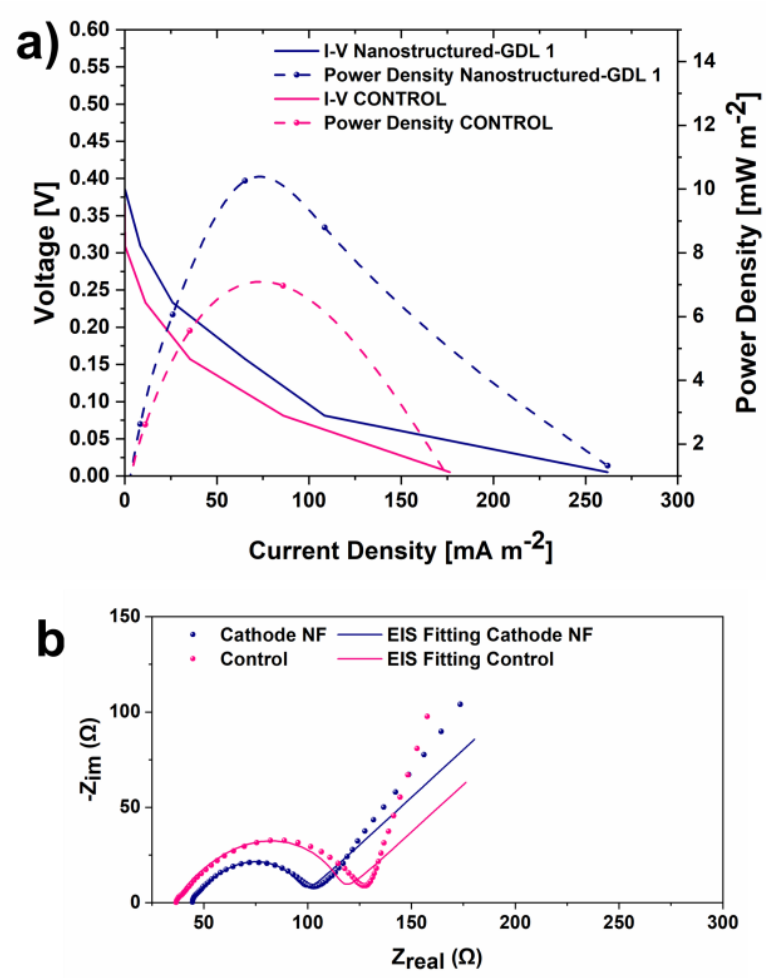

Fig. 3. a) Polarization curves obtained by LSV characterization. Potential vs. current density curves (left axis, straight lines) and power density vs. current density (right axis, dash lines) curves of SCMFCs with nanostructured-GDL and commercial GDL, used as reference. b) Typical impedance spectra of nanostructured-GDL (blue dot and line) and commercial-GDL (pink dot and line).

As highlighted in Fig.3b), all SCMFCs show a similar value of series resistance $\mathrm{R} 1$, independently of cathode electrodes. This is to be expected, since electrolyte, wires and electrical connection are identical for all SCMFCs. Moreover, the lower is the charge transfer at the electrode/electrolyte interface R3, higher is the capacity of cathode electrode to ensure a faster electrons flow. This result demonstrates the effectiveness of nanostructured-GDL to ensure an enhancement of oxygen diffusion, improving consequently the occurring of direct-ORR and the overall SCMFCs performance. A similar trend is observed for the transport resistance R2, which is visible in the high-frequency smaller arc sketched in fig.3b). A lower R2 defines an increasing of electrode transport properties and since all other aspects of cathode electrodes are the same, it is possible to confirm how nanostructured-GDL results to be more efficient in carrying out ORR. 
Table 1. Typical resistance values ( $R 1, R 2$ and $R 3$ ) calculated from the fitting procedure on Electrochemical Impedance Spectroscopy (EIS) data. For each parameter, the maximum variation observed between three nominally identical Microbial Fuel Cells (MFCs) was 10\%.

\begin{tabular}{|c|c|c|c|}
\hline \multirow{2}{*}{$\begin{array}{c}\text { Cathode } \\
\text { electrodes }\end{array}$} & $\mathrm{R} 1$ & $\mathrm{R} 2$ & $\mathrm{R} 3$ \\
\cline { 2 - 4 } & {$[\Omega]$} & {$[\Omega]$} & {$[\Omega]$} \\
\hline $\begin{array}{c}\text { Nanostructure } \\
\text { d-GDL }\end{array}$ & 44.3 & 12.04 & 38.4 \\
\hline $\begin{array}{c}\text { Commercial- } \\
\text { GDL }\end{array}$ & 36.93 & 25.69 & 50.45 \\
\hline
\end{tabular}

Moreover, in line with all obtained results, the analysis performed in terms of energy recovery parameter, defined as ratio of generated power integral and the internal volume of devices, allow evaluating the overall SCMFC performance. SCMFCs with a nanostructured-GDL showed an energy recovery equal to $60.83 \mathrm{~mJ} \mathrm{~m}-3$, which was one order of magnitude higher than the one obtained with commercial-GDL, close to $3.92 \mathrm{~mJ} \mathrm{~m}-3$

\section{Conclusion}

In the present work, nanostructured-GDL was designed as new gas diffusion layer to improve the oxygen diffusion inside SCMFCs, exploiting the intrinsic properties of nanofibers, such as high porosity, high surface area to volume ratio and light weight. Moreover, through electrospinning process, a direct deposition of nanofiber mats can be collected onto carbon based materials, used as cathode electrodes, without the necessity of binder to bond GDL with the carbon backbone. Morphological properties confirm a higher porosity obtained with nanostructured-GDL, than the one reached when commercial-GDL is applied as reference results. Moreover, all obtained results demonstrate that nanostructured-GDL play a pivotal role to improve all SCMFCs performance. Indeed, SCMFCs with nanostructured-GDL reach a maximum current density which is double of the value achieved when commercial
GDL is analyzed. Since all other aspects are in common between all cathode electrodes, it is possible to confirm that all difference can be addicted to the presence of nanofiber mats as GDL. All obtained results demonstrate the effectiveness of nanostructured-GDL to ensure an enhancement of oxygen diffusion, improving consequently the occurring of direct-ORR and the overall SCMFCs performance.

\section{Reference}

[1] B. Walsh, P. Ciais, I.A. Janssensz,; J.Penuelas, J, K. Riahi, F. Rydza, D.P. Van Vuuren, M. Obersteiner. Nature Communications (2017), 8, 14856-68

[2] Logan, B.E.; Rabaey, K. Science (2012), 337, 686690

[3] Logan, B.E. Microbial Fuel Cells, New York: John Wiley \& Sons; 2008

[4] Logan, B.E.; Hamelers, B.; Rozendal, R.; Schroder, U.; Keller, J.; Freguia, S.; Aelterman, P.; Verstraete, W.; Rabaey, K. Environmental Science and Technology (2006), 40, 5181-92

[5] Babauta, J.; Renslow, R.; Lewandowski, Z.; Beyenal, H.. Biofouling (2012), 28, 789-812

[6] Harnisch, F.; Aulenta, F.; Schroeder, U. Comprehensive Biotechnology 2nd ed; Elsevier: Amsterdam, 2011, p. 644-659

[7] Santoro C, Stradlhofer A, Hacker V, Squadrito G, Schroder U, Li B. Journal of Power Sources (2013); 243:499-507

[8] Oh S, Min B, Logan BE. Environmental Science Technology (2004); 38: 4900-4904

[9] Cheng S, Liu H, Logan BE. Electrochemistry communications 2006; 8:489-494

[10] Massaglia G, Chiodoni A, Salvador GP, Delmondo L, Munoz-Tabares JA, Bocchini S, Sacco A, Bianco S, Saracco G, Quaglio M. RSC Adv. (2017);7:38812-38818 [11] Massaglia G., Margaria V., Sacco A., Castellino M., Chiodoni A., C.F. Pirri, Quaglio M., IJHE 44 (2019) : 4442-9

[12] Jackson A, Strickler A, Higgins D, Jaramillo TF.. Nanomaterials (2018); 8: 38-53 